MATHEMATICS OF COMPUTATION

Volume 72, Number 244, Pages 2071-2076

S 0025-5718(03)01489-3

Article electronically published on February 3, 2003

\title{
NEW AMICABLE FOUR-CYCLES
}

\author{
KARSTEN BLANKENAGEL, WALTER BORHO, AND AXEL VOM STEIN
}

ABSTRACT. Fifty new amicable four-cycles are discovered by the constructive method invented in 1969 by the second author.

1. Let $\tau(n)$ denote the sum of proper divisors of a natural number $n$, and let $\sigma(n)=n+\tau(n)$. We consider when the sequence $n, \tau(n), \tau^{(2)}(n):=\tau(\tau(n)), \ldots$ becomes periodic. If $n=\tau^{(k)}(n)$ with $k$ minimal, then $n_{1}=n, n_{2}=\tau(n), n_{3}=$ $\tau^{(2)}(n), \ldots, n_{k}=\tau^{(k-1)}(n)$ is called an amicable $k$-cycle. The study of amicable 1-cycles (perfect numbers) and of amicable 2-cycles (amicable pairs) has a thousandyear-old history. Here we study amicable four-cycles. The smallest example is

$$
\begin{array}{ll}
n_{1}=2^{2} \cdot 5 \cdot 17 \cdot 3719, & n_{3}=2^{2} \cdot 521 \cdot 829, \\
n_{2}=2^{2} \cdot 5 \cdot 193 \cdot 401, & n_{4}=2^{5} \cdot 40787,
\end{array}
$$

discovered by H. Cohen [5] in 1970 by an exhaustive trial and error search below $60,000,000$.

2. Alternatively, one may try to construct amicable four-cycles of a special form. This can be done by means of the following theorem, due to the second author.

Theorem 1 (3). Let $a_{1}$ and $a_{2}$ be natural numbers, $a_{1} \neq a_{2}$, and let $D:=$ $a_{1} a_{2}-\tau\left(a_{1}\right) \tau\left(a_{2}\right)$. Let $d_{1} d_{2}=a_{1} a_{2}$ be any factorization into two natural numbers $d_{1}, d_{2}$. Consider the six numbers $p_{i j}, r_{i}(i, j=1,2)$

$$
\begin{aligned}
p_{i j} & :=\frac{1}{D}\left(\tau\left(a_{i+1}\right) \sigma\left(a_{i}\right)+d_{j} \sigma\left(a_{i+1}\right)\right), \quad \text { where } a_{3}:=a_{1}, \\
r_{i} & :=\frac{1}{a_{i}} \tau\left(a_{i} p_{i 1} p_{i 2}\right) .
\end{aligned}
$$

If all six are primes, and $p_{i j} \nmid a_{i}, r_{i} \nmid a_{i}, p_{i 1} \neq p_{i 2}(i, j=1,2)$, then the following is an amicable four-cycle:

$$
\begin{array}{ll}
n_{1}=a_{1} p_{11} p_{12}, & n_{3}=a_{2} p_{21} p_{22}, \\
n_{2}=a_{1} r_{1}, & n_{4}=a_{2} r_{2} .
\end{array}
$$

The smallest example is

$$
\begin{array}{ll}
n_{1}=3^{3} \cdot 5 \cdot 7 \cdot 83 \cdot 359, & n_{3}=3^{3} \cdot 5 \cdot 11 \cdot 79 \cdot 263, \\
n_{2}=3^{3} \cdot 5 \cdot 7 \cdot 31643, & n_{4}=3^{3} \cdot 5 \cdot 11 \cdot 20183,
\end{array}
$$

which was found in [3] in 1969 without use of a computer.

Received by the editor September 19, 2001 and, in revised form, February 18, 2002.

2000 Mathematics Subject Classification. Primary 11A25.

Key words and phrases. Number theory, sociable numbers. 
To specify these four numbers, it is enough to give $a_{1}, a_{2}$ and $d_{1}$, because the rest is then given by formulas (11) and (2) of the theorem. In the example

$$
3^{3} \cdot 5 \cdot\left\{\begin{array}{l}
7=a_{1}, \\
11=a_{2},
\end{array} \quad d_{1}=3^{2} \cdot 5 \cdot 7\right.
$$

3. By a constructive search based on the above theorem, we produced 50 new (amicable) four-cycles specified below. Of the 60 (amicable) four-cycles previously known, 30 were found by this same constructive method, while 30 were found by trial and error (below $10^{12}$ ). Here is the list of the discoverers:

\begin{tabular}{|llcll|}
\hline years & Discoverers & $\begin{array}{c}\text { number of } \\
\text { discoveries }\end{array}$ & method & references \\
\hline 1969 & Borho & 1 & constructive & {$[3]$} \\
1970 & Cohen & 8 & trial and error & {$[5]$} \\
1972 & David / Root & $4 / 5$ & trial and error & {$[6] /[2]$} \\
1990 & Yuanhua & 3 & constructive & {$[10]$} \\
1990 & Flammenkamp & 7 & trial and error & {$[7]$} \\
$1990-95$ & Moews, Moews & 9 & trial and error & {$[8] /[9]$} \\
$1990-98$ & Moews, Moews & 8 & constructive & {$[8]$} \\
$1997-00$ & Pedersen & 9 & constructive & \\
2000 & Baader & 1 & trial and error & \\
$2000-01$ & Ren Yuanhua & 9 & constructive & \\
$2000-01$ & new & 50 & constructive & \\
\hline
\end{tabular}

4. In the constructive search with the theorem above, the choice of $a_{1}$ and $a_{2}$ is of course essential. It is useful to check the following necessary condition for $a_{1}, a_{2}$.

Theorem 2 (Four-cycle condition). In order that the $p_{i j}$ in Theorem 1 can be integers, it is necessary that

$$
\left(a_{1}-a_{2}\right) f g \equiv 0 \bmod D,
$$

where $f$ resp. $g$ is the greatest common divisor of $\sigma\left(a_{1}\right), \sigma\left(a_{2}\right)$ resp. of $a_{1}, a_{2}$.

We call a four-cycle given by Theorem 1 "regular of type $(r, s)$ " if $a_{1}, a_{2}$ have the form

$$
a_{1}=a p_{1} p_{2} \ldots p_{r}, \quad a_{2}=a q_{1} q_{2} \ldots q_{s},
$$

with some greatest common divisor $a$, and different primes $p_{1}, \ldots, p_{r}, q_{1}, \ldots, q_{s}$ not dividing $a$. The 50 new amicable four-cycles discovered here are all "regular" in this sense. Nonregular or "exotic" examples were found by Ren Yuanhua and Pedersen.

5. For our constructive search for four-cycles, we must make the denominator $D:=D\left(a_{1}, a_{2}\right)=a_{1} a_{2}-\tau\left(a_{1}\right) \tau\left(a_{2}\right)=a_{1} \sigma\left(a_{2}\right)+a_{2} \sigma\left(a_{1}\right)-\sigma\left(a_{1}\right) \sigma\left(a_{2}\right)$ small in order to increase the chance that the $p_{i j}$ become integers. But we can cancel by $g_{i}:=\operatorname{gcd}\left(a_{i}, \sigma\left(a_{i}\right)\right)$ for $i=1,2$, and in addition by $\operatorname{gcd}\left(\sigma\left(a_{1}\right) / g_{1}, \sigma\left(a_{2}\right) / g_{2}\right)$. Let 
$\bar{D}\left(a_{1}, a_{2}\right)$ denote the result. What we really have to make small is this $\bar{D}\left(a_{1}, a_{2}\right)$. (One might take $d_{j}$ a multiple of $g_{j}$ to make $p_{i j}$ integer.)

Let us describe, for example, our search for regular four-cycles of type $(1,1)$. So let $a_{1}=a p_{1}, a_{2}=a q_{1}$ as in section 4 . For $a$ we took all values $\leq 50,000,000$ with $\bar{D}(a, a)<100$. These are 6224 numbers. Next, $p_{1}$ must be chosen greater than $\tau(a) \sigma(a) / D(a, a)$ (to make $D\left(a p_{1}, a\right)$ positive). All primes $p_{1}$ greater than this lower bound were taken in increasing order as long as $\bar{D}\left(a p_{1}, a\right)<100$. Finally, $q_{1}$ was chosen in such a way that the four-cycle condition (Theorem 2) was satisfied. To all pairs $a_{1}=a p_{1}, a_{2}=a q_{1}$ so chosen, Theorem 11 was applied; that is, all factorizations $d_{1} d_{2}=a_{1} a_{2}$ were checked to see whether they give an amicable four-cycle.

This search produced seven of the thirteen known four-cycles of type $(1,1)$, plus in addition two new ones, numbers 2), 3) of the list below.

Searches for four-cycles of types $(1,0),(2,1),(2,2),(3,1),(3,2),(3,3)$ were performed in a similar manner. The new results are listed below. Note that no four-cycles of type $(1,0)$ are known so far. It is an interesting open problem to find a four-cycle of this type.

An interesting phenomenon that occurs so far only in four-cycles of type $(1,1)$ is that $p_{11}$ may equal $p_{21}$. This actually occurs in 9 of the 16 known cases of type $(1,1)$.

For our search for amicable four-cycles we spent a total of about 3 years of computation on Sun desk calculators of $333 \mathrm{MHz}$.

6. The following table specifies all 50 new amicable four-cycles found by the searches described above. In fact, for each of the 50 four-cycles we give the triples $a_{1}, a_{2}$ and $d_{1}$ in prime factorization. From these data, the full four-cycle itself can be easily computed by the formulae given in Theorem 11 For instance, for four-cycle number 4 below, one obtains the 14 decimal digit numbers (in prime decomposition):

$$
\begin{aligned}
& n_{1}=2 \cdot 7 \cdot 223 \cdot 5 \cdot 11 \cdot 3121 \cdot 73589, \quad n_{3}=2 \cdot 7 \cdot 223 \cdot 23 \cdot 4013 \cdot 215417, \\
& n_{2}=2 \cdot 7 \cdot 223 \cdot 5 \cdot 11 \cdot 288231067, \quad n_{4}=2 \cdot 7 \cdot 223 \cdot 23 \cdot 689238587 .
\end{aligned}
$$

We let each line of the following table begin with an ordinal number of the corresponding four-cycle, followed by the number of decimal digits of the smallest member of the four-cycle, with a "D" for "decimal digits". Next, $a_{1}$ and $a_{2}$ are given,

with the common divisor given only once. Finally, $d_{1}$ is given. The four-cycles are ordered according to their type.
type $(1,1)$
1) $23 \mathrm{D}$
$2 \cdot 7^{2} \cdot 13 \cdot 19 \cdot 101 \cdot\left\{\begin{array}{l}118169 \\ 236339\end{array}\right.$
$d_{1}=2 \cdot 7^{2} \cdot 13 \cdot 19 \cdot 101$
2) $26 D$
$3^{2} \cdot 5^{2} \cdot 19 \cdot 29 \cdot 41 \cdot\left\{\begin{array}{l}491 \\ 294461\end{array}\right.$
$d_{1}=3^{2} \cdot 5^{2} \cdot 19 \cdot 41 \cdot 294461$
3) $27 D$
$3^{3} \cdot 7^{2} \cdot 13 \cdot 17 \cdot 67 \cdot\left\{\begin{array}{l}179 \\ 49139\end{array}\right.$
$d_{1}=3^{3} \cdot 7^{2} \cdot 13 \cdot 17 \cdot 67$ 

type $(2,1)$
4) $14 D \quad 2 \cdot 7 \cdot 223 \cdot\left\{\begin{array}{l}5 \cdot 11 \\ 23\end{array}\right.$
$d_{1}=2 \cdot 7 \cdot 223$
5) $15 D \quad 3^{3} \cdot 5^{3} \cdot\left\{\begin{array}{l}7 \cdot 59 \\ 89\end{array}\right.$
$d_{1}=3^{3} \cdot 5^{3}$
6) $16 D \quad 3^{2} \cdot 5^{2} \cdot 31 \cdot\left\{\begin{array}{l}7 \cdot 59 \\ 89\end{array}\right.$
$d_{1}=3^{2} \cdot 5^{2} \cdot 31$
7) $17 \mathrm{D} \quad 3^{2} \cdot 5 \cdot 13 \cdot 19 \cdot\left\{\begin{array}{l}53 \cdot 379 \\ 71\end{array}\right.$
$d_{1}=3^{2} \cdot 5 \cdot 13 \cdot 19 \cdot 53$
8) $22 D \quad 3^{5} \cdot 5 \cdot 13 \cdot\left\{\begin{array}{l}17 \cdot 109 \\ 17929\end{array}\right.$
$d_{1}=3^{7} \cdot 5 \cdot 13 \cdot 109$
9) $23 D \quad 3^{5} \cdot 7^{2} \cdot 13 \cdot 83 \cdot\left\{\begin{array}{l}11 \cdot 53 \\ 1871\end{array}\right.$
$d_{1}=3^{5} \cdot 7^{2} \cdot 13 \cdot 1871$
10) $24 D \quad 3^{2} \cdot 5 \cdot 13 \cdot 23 \cdot 137 \cdot\left\{\begin{array}{l}29 \cdot 547 \\ 349\end{array}\right.$
$d_{1}=3^{2} \cdot 5 \cdot 13 \cdot 23 \cdot 29 \cdot 137$
11) $24 D \quad 2^{4} \cdot 101 \cdot\left\{\begin{array}{l}29 \cdot 797 \\ 113\end{array}\right.$
$d_{1}=2^{4} \cdot 29$
12) $26 D \quad 3^{7} \cdot 5^{2} \cdot 41 \cdot\left\{\begin{array}{l}11 \cdot 79 \\ 7529\end{array}\right.$
$d_{1}=3^{11} \cdot 5^{2} \cdot 41$
13) $31 D \quad 3^{2} \cdot 5 \cdot 13 \cdot 19 \cdot\left\{\begin{array}{l}571 \cdot 162749 \\ 29\end{array}\right.$
$d_{1}=3^{2} \cdot 5 \cdot 13 \cdot 19 \cdot 571$
14) $32 D \quad 3^{4} \cdot 7^{2} \cdot 11 \cdot 19 \cdot\left\{\begin{array}{l}379 \cdot 523 \\ 5177119\end{array}\right.$
$d_{1}=3^{4} \cdot 11 \cdot 19 \cdot 5177119$
15) $36 D \quad 2^{8} \cdot 619 \cdot\left\{\begin{array}{l}1487 \cdot 105229 \\ 391455599\end{array}\right.$
$d_{1}=2^{8} \cdot 619 \cdot 105229$
16) $36 D \quad 3^{2} \cdot 7 \cdot 11 \cdot 17 \cdot 23 \cdot\left\{\begin{array}{l}101 \cdot 397493 \\ 2039\end{array}\right.$
$d_{1}=3^{2} \cdot 7 \cdot 17 \cdot 23^{2} \cdot 101$
type $(2,2)$
17) $16 D \quad 3^{2} \cdot 5 \cdot 13 \cdot\left\{\begin{array}{l}11 \cdot 1279 \\ 23 \cdot 127\end{array}\right.$
$d_{1}=3^{2} \cdot 5 \cdot 13 \cdot 1279$
18) $17 D \quad 2^{3} \cdot\left\{\begin{array}{l}13 \cdot 3079 \\ 19 \cdot 293\end{array}\right.$
$d_{1}=2^{3} \cdot 13$
19) $19 D \quad 3^{4} \cdot 11^{2} \cdot 17 \cdot\left\{\begin{array}{l}5 \cdot 373 \\ 13 \cdot 43\end{array}\right.$
$d_{1}=3^{4} \cdot 11^{2} \cdot 13 \cdot 17 \cdot 43$
20) $20 D \quad 2 \cdot 5 \cdot 13 \cdot\left\{\begin{array}{l}29 \cdot 139 \\ 47 \cdot 2099\end{array}\right.$
$d_{1}=2 \cdot 5 \cdot 13 \cdot 47 \cdot 139$
21) $21 D \quad 3^{4} \cdot 7^{2} \cdot 11^{2} \cdot\left\{\begin{array}{l}17 \cdot 97 \\ 41 \cdot 1049\end{array}\right.$
$d_{1}=3^{5} \cdot 7^{2} \cdot 11^{2} \cdot 1049$
22) $22 D \quad 3^{3} \cdot 7^{2} \cdot 13 \cdot\left\{\begin{array}{l}11 \cdot 199 \\ 17 \cdot 1559\end{array}\right.$
$d_{1}=3^{3} \cdot 7^{2} \cdot 11 \cdot 13 \cdot 199$
23) $22 D \quad 2^{6} \cdot\left\{\begin{array}{l}107 \cdot 2099 \\ 179 \cdot 2939\end{array}\right.$
$d_{1}=2^{5} \cdot 179 \cdot 2099$
24) $24 D \quad 2^{5} \cdot 97 \cdot\left\{\begin{array}{l}193 \cdot 2447 \\ 197 \cdot 2909\end{array}\right.$
$d_{1}=2^{5} \cdot 97 \cdot 197 \cdot 2447$ 


$$
\begin{aligned}
& \text { 25) } 25 D \quad 3^{2} \cdot 5^{3} \cdot 13^{2} \cdot\left\{\begin{array}{l}
29 \cdot 193 \\
103 \cdot 14549
\end{array} \quad d_{1}=3^{2} \cdot 5^{3} \cdot 13^{2} \cdot 103 \cdot 193\right. \\
& \text { 26) } 25 D \quad 3^{3} \cdot 5 \cdot 11 \cdot 43 \cdot\left\{\begin{array}{l}
101 \cdot 1031 \\
251 \cdot 1289
\end{array} \quad d_{1}=3^{3} \cdot 5 \cdot 11 \cdot 43 \cdot 101 \cdot 1289\right. \\
& \text { 27) } 25 D \quad 3^{2} \cdot 5 \cdot 13 \cdot 41 \cdot\left\{\begin{array}{l}
19 \cdot 233 \\
29 \cdot 2347
\end{array} \quad d_{1}=3^{2} \cdot 5 \cdot 13 \cdot 41^{2} \cdot 233\right. \\
& \text { 28) } 26 D \quad 3 \cdot 5 \cdot 7 \cdot 19 \cdot\left\{\begin{array}{l}
13 \cdot 509 \\
577 \cdot 4787
\end{array} \quad d_{1}=3 \cdot 5 \cdot 7 \cdot 19 \cdot 509\right. \\
& \text { 29) } 26 D \quad 3^{2} \cdot 7 \cdot 11 \cdot 13 \cdot 43 \cdot\left\{\begin{array}{l}
101 \cdot 1031 \\
251 \cdot 1289
\end{array} \quad d_{1}=3^{2} \cdot 7 \cdot 11 \cdot 13 \cdot 43 \cdot 101 \cdot 1289\right. \\
& \text { 30) } 28 D \quad 3^{3} \cdot 5^{4} \cdot 19 \cdot\left\{\begin{array}{l}
37 \cdot 239 \\
47 \cdot 9803
\end{array} \quad d_{1}=3^{4} \cdot 5^{5} \cdot 19 \cdot 47\right. \\
& \text { 31) } 28 D \quad 2^{6} \cdot\left\{\begin{array}{l}
79 \cdot 2699 \\
367 \cdot 23819
\end{array} \quad d_{1}=2^{6} \cdot 367 \cdot 2699\right. \\
& \text { 32) } 30 D \quad 3^{4} \cdot 5 \cdot 11 \cdot\left\{\begin{array}{l}
23 \cdot 11699 \\
419 \cdot 3167
\end{array} \quad d_{1}=3^{4} \cdot 5 \cdot 11 \cdot 23\right. \\
& \text { 33) } 30 D \quad 2^{2} \cdot 11 \cdot 79 \cdot\left\{\begin{array}{l}
17 \cdot 13903 \\
89 \cdot 112507
\end{array} \quad d_{1}=2^{2} \cdot 11 \cdot 79 \cdot 13903\right. \\
& \text { 34) } 31 D \quad 3^{3} \cdot 5^{3} \cdot 13 \cdot\left\{\begin{array}{l}
199 \cdot 4751 \\
271 \cdot 336599
\end{array} \quad d_{1}=3^{3} \cdot 5^{3} \cdot 13 \cdot 271\right. \\
& \text { 35) } 31 D \quad 3^{2} \cdot 5^{2} \cdot 13 \cdot 31 \cdot\left\{\begin{array}{l}
199 \cdot 4751 \\
271 \cdot 336599
\end{array} \quad d_{1}=3^{2} \cdot 5^{2} \cdot 13 \cdot 31 \cdot 271\right. \\
& \text { 36) } 37 \mathrm{D} \quad 3^{2} \cdot 5 \cdot 13 \cdot 23 \cdot\left\{\begin{array}{l}
19 \cdot 101429 \\
461 \cdot 22271129
\end{array} \quad d_{1}=3^{2} \cdot 5 \cdot 13 \cdot 19 \cdot 23 \cdot 101429\right. \\
& \text { 37) } 41 D \quad 3^{2} \cdot 5 \cdot 11 \cdot 19 \cdot\left\{\begin{array}{l}
131 \cdot 5651377 \\
509 \cdot 30557
\end{array} \quad d_{1}=3^{2} \cdot 5 \cdot 11 \cdot 19 \cdot 131\right. \\
& \text { type }(3,1) \\
& \text { 38) } 32 D \quad 3^{2} \cdot 7^{2} \cdot 11 \cdot 13 \cdot\left\{\begin{array}{l}
71 \cdot 83 \cdot 2851 \\
245519
\end{array} \quad d_{1}=3^{2} \cdot 7^{2} \cdot 11 \cdot 13 \cdot 71 \cdot 83\right. \\
& \text { 39) } 48 \mathrm{D} \quad 2 \cdot 5 \cdot 11^{2} \cdot 241 \cdot\left\{\begin{array}{l}
127 \cdot 181 \cdot 70853 \\
434571199
\end{array} d_{1}=2 \cdot 5 \cdot 11^{2} \cdot 241\right. \\
& \text { type }(3,2) \\
& \text { 40) } 17 D \quad 2^{3} \cdot\left\{\begin{array}{l}
13 \cdot 23 \cdot 109 \\
167 \cdot 1231
\end{array} \quad d_{1}=2^{3} \cdot 23 \cdot 167\right. \\
& \text { 41) } 19 \mathrm{D} \quad 3^{3} \cdot 5^{2} \cdot\left\{\begin{array}{l}
11 \cdot 29 \cdot 167 \\
23 \cdot 449
\end{array} \quad d_{1}=3^{3} \cdot 5^{2} \cdot 11 \cdot 23 \cdot 29\right. \\
& \text { 42) } 24 D \quad 2^{2} \cdot 11 \cdot\left\{\begin{array}{l}
17 \cdot 439 \cdot 503 \\
31 \cdot 11087
\end{array} \quad d_{1}=2^{2} \cdot 11 \cdot 439 \cdot 503\right. \\
& \text { 43) } 25 D \quad 3^{2} \cdot 5^{3} \cdot 13 \cdot\left\{\begin{array}{l}
29 \cdot 59 \cdot 389 \\
149 \cdot 8423
\end{array} \quad d_{1}=3^{2} \cdot 5^{3} \cdot 13 \cdot 29 \cdot 389\right. \\
& \text { 44) } 35 D \quad 3^{2} \cdot 7^{2} \cdot 11 \cdot 13 \cdot\left\{\begin{array}{l}
97 \cdot 109 \cdot 569 \\
197 \cdot 1667959
\end{array} \quad d_{1}=3^{2} \cdot 7^{2} \cdot 11 \cdot 13 \cdot 109 \cdot 569\right. \\
& \text { 45) } 40 D \quad 3^{5} \cdot 5 \cdot 13 \cdot\left\{\begin{array}{l}
17 \cdot 263 \cdot 67103 \\
197 \cdot 122039807
\end{array} \quad d_{1}=3^{5} \cdot 5 \cdot 13 \cdot 122039807\right.
\end{aligned}
$$




$$
\begin{aligned}
& \text { 46) } 42 D \quad 2^{5} \cdot\left\{\begin{array}{l}
47 \cdot 2137 \cdot 6323 \\
101 \cdot 642640039
\end{array} \quad d_{1}=2^{5} \cdot 2137 \cdot 6323\right. \\
& \text { 47) } 47 \mathrm{D} \quad 2 \cdot 5 \cdot 11 \cdot\left\{\begin{array}{l}
239 \cdot 1051 \cdot 2763119 \\
31 \cdot 181324319039
\end{array} \quad d_{1}=2 \cdot 5 \cdot 31 \cdot 239 \cdot 1051 \cdot 2763119\right. \\
& \text { 48) } 52 D \quad 2^{2} \cdot 11 \cdot 149 \cdot\left\{\begin{array}{l}
13 \cdot 1297 \cdot 15074627 \\
167 \cdot 272306275957
\end{array} d_{1}=2^{2} \cdot 11 \cdot 149 \cdot 272306275957\right. \\
& \text { type }(3,3) \\
& \text { 49) } 28 D \quad 2^{3} \cdot\left\{\begin{array}{l}
19 \cdot 23 \cdot 47 \\
79 \cdot 251 \cdot 31727
\end{array} \quad d_{1}=2^{3} \cdot 23 \cdot 79 \cdot 251\right. \\
& \text { 50) } 45 D \quad 2^{4} \cdot\left\{\begin{array}{l}
23 \cdot 83 \cdot 227 \\
191 \cdot 15791 \cdot 10112749
\end{array} \quad d_{1}=2^{4} \cdot 83\right.
\end{aligned}
$$

7. The two four-cycles 1), 13) were found by a different type of search: $a_{1}, a_{2}$ were chosen as so-called breeders for amicable number pairs, as studied in [4, [1. These two new cycles are due to all three authors; the other ones are due to the first two authors. A complete list of all 110 known amicable four-cycles is provided by J. O. M. Pedersen on his internet amicable.adsl.dk pages

\section{http://amicable.adsl.dk/aliquot/sociable.txt. \\ REFERENCES}

1. S. Battiato and W. Borho, Breeding amicable numbers in abundance II, Math. Comp. 70, (2001), 1329-1333. MR 2002b:11011

2. M. Beeler, R. W. Gosper and R. Schroeppel, M.I.T. Artificial Intelligence Memo \#239, (29.02.72), Item 61.

3. W. Borho, Über die Fixpunkte der k-fach iterierten Teilersummenfunktion, Mitt. Math. Gesellsch. Hamburg 9, (1969), 34-48. MR 40:7189

4. W. Borho and H. Hoffmann, Breeding amicable numbers in abundance, Math. Comp. 46, (1986), 281-293. MR 87c:11003

5. H. Cohen, On amicable and sociable numbers, Math. Comp. 24, (1970), 423-429. MR 42:5887

6. J.S. Devitt, R. K. Guy and J.L. Selfridge, Third report on aliquot sequences, Congr. Numer. XVIII, Proc. 6th Manitoba Conf. Numerical Math., (1976), 177-204. MR 80d:10001

7. A. Flammenkamp, New sociable numbers, Math. Comp. 56, (1991), 871-873. MR 92a:11004

8. D. Moews and P. C. Moews, A search for aliquot cycles below $10^{10}$, Math. Comp. 57, (1991), 849-855. MR 92e:11151

9. D. Moews and P. C. Moews, A search for aliquot cycles and amicable pairs, Math. Comp. 61, (1993), 935-938. MR 94a:11198

10. Ren Yuanhua, On constructing sociable numbers, (1990), preprint.

Karsten Blankenagel, BUGH FB 7, Gaussstrasse 20, 42097 Wuppertal, Germany

E-mail address: kblanken@math.uni-wuppertal.de

Walter Borho, Bugh FB 7, Gaussstrasse 20, 42097 Wuppertal, Germany

E-mail address: Walter.Borho@math.uni-wuppertal.de

Axel vom Stein, BUGH FB 7, Gaussstrasse 20, 42097 Wuppertal, Germany

E-mail address: vomstein@uni-wuppertal.de 